\title{
Assessment of Adverse Effects of Most Commonly Prescribed Anticancer Drugs in a Tertiary Care Teaching Hospital
}

\author{
Salu Sunny ${ }^{1 *}$, Athira Thampi ${ }^{1}$, Johnkennedy ${ }^{1}$, Nishitha Shetty ${ }^{2}$, Sajeev Kumar Babasahib ${ }^{3}$, Chinju \\ Chacko4.
}

${ }^{1}$ Department of Pharmacy Practice, Karavali College of Pharmacy, Mangalore, Karnataka, INDIA.

${ }^{2}$ Department of Medical Oncology, Father Muller Medical College Hospital, Mangalore, Karnataka, INDIA.

${ }^{3}$ Department of Pharmaceutics, Ahalia School of Pharmacy, Palakkad, Kerala, INDIA.

${ }^{4}$ Department of Pharmacy Practice, NGSM Institute of Pharmaceutical Sciences, Deralakatte, Mangalore, Karnataka, INDIA.

\begin{abstract}
Background: Chemotherapeutic agents account for high inclination towards adverse drug reactions due to its narrow therapeutic window, increased toxicity and highly complex regimen making pharmacovigilance studies essential in oncology. Aim:The aim of the study is to assess the adverse effects of most commonly prescribed anticancer drugs in a tertiary care teaching hospital and to evaluate its causality, severity and preventability. Materials and methods: A prospective observational study was conducted on 200 patients taking chemotherapy in oncology unit. Patient information was obtained using a data collection form and data was collected using a structured questionnaire. Assessment of adverse drug reactions were analyzed using WHO - UMC causalilty assessment scale, Hartwig -Siegel severity assessment scale and modified schumock and thornton preventability scale. Results: Cyclophosphamide (10.62\%) was the most commonly prescribed anticancer drug. A total of 450 ADRs were observed from 109 patients. Females $(60.55 \%)$ were more prone to ADRs than males. The most frequent adverse reaction was vomiting $(10 \%)$ followed by anaemia $(9.11 \%)$, alopecia $(7.11 \%)$, fatigue $(6.67 \%)$. WHO causality assessment scale showed $45.78 \%$ of the reactions to be "possible", $40.44 \%$ to be "probable", $12.89 \%$ to be "unlikely" and $0.89 \%$ to be "certain". Modified Hartwig and Siegel scale showed most reactions, $54.13 \%$ to be mild, $44.95 \%$ to be moderate and $0.92 \%$ to be severe reactions. According to modified Schumock and Thornton scale, $46.22 \%$ of the ADRs were "definitely preventable", $20.67 \%$ were "probably preventable" and $33.11 \%$ were "not preventable". Conclusion: Chemotherapeutic agents are highly associated with adverse reactions. Measures should be undertaken to improve ADR monitoring and reporting, thereby decrease the incidence of ADR, improve patient's quality of life and ensure safe use of drugs.
\end{abstract}

Key words: Chemotherapy, Adverse drug reactions, Causality, Severity, Preventability.

\section{INTRODUCTION}

An adverse drug reaction (ADR) is any undesirable or accidental outcome of drug administration that has a foremost role in rising economic burden and sufferings by the patients. The World Health Organization (WHO) defines an ADR as "any response to a drug, which is noxious, unintended and occurs at doses used in man for prophylaxis, diagnosis or therapy". ${ }^{1}$ Monitoring, reporting and documentation of ADRs are necessary to support and certify safe use of drugs. Recent epidemiological studies estimated ADRs to be the fourth to sixth leading cause of death. ${ }^{2}$ It is vital to identify ADRs as early as possible and prevent them if possible, to ensure the wellbeing of the patient at a sensible cost.

The WHO realized the importance of having an efficient, dynamic surveillance system to monitor the occurrence of ADRs, which was the basis for starting the International Drug Monitoring Program, thereby, the Pharmacovigilance program was started in India in 2010 with the objectives of monitoring drug safety and creating an ADR
DOI: 10.5530/ijopp.10.4.55

Address for correspondence: Salu Elesabath Sunny, Pharm D Intern, Karavali College of Pharmacy, NH. 13, Vamanjoor, Mangalore - 575028, D.K. Dist., Karnataka State, INDIA. Phone no: 9447929580 Email Id: salusunny109@gmail. com

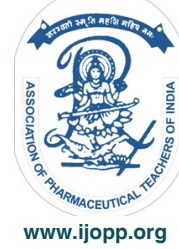


database for our population. ${ }^{3}$

The extend of adverse drug reactions suffered by oncology patients is immense since chemotherapy regimens are highly complex and cancer patients are the liable population with little tolerance. Anticancer drug therapies are more susceptible to cause ADRs as these agents are cytotoxic and can damage the normally dividing cells along with cancerous cells, leading to various side effects. Chemotherapeutic agents have a narrow therapeutic index, where a small difference in the dose can be noxious to the rapidly dividing cells like bone marrow, gastrointestinal tract and hair follicles. Also, patients on chemotherapy will be on multi drug treatments making them at risk to ADRs.

Risks associated with drug use can be recognised and assessed with the help of hospital based ADR monitoring and reporting programs. Such programs can be a helping hand for the prescribers to spot ADRs and deal with them more professionally and efficiently.

Pharmacists must play a crucial role in developing, maintaining and evaluating a program to reduce the risk of ADRs through detecting, reporting and assessing any suspected ADR. Reporting of ADR and its management will improve the efficiency of therapy and decrease the adverse effects and eventually reduces mortality.

\section{MATERIALS AND METHODS}

A prospective observational study was conducted at the oncology department of Father Muller Medical College Hospital, Mangalore for a period of 4 months from November 2016 to February 2017 after seeking approval from institutional ethics committee (FMMC/ FMIEC/ 3046/ 2016). A total of 200 patients were included in the study. Cancer patients of all age groups admitted in oncology ward, who had undergone at least one cycle of chemotherapy, were incorporated, while patients taking radiotherapy were excluded. Patient information was collected and entered in data collection form. An adverse drug reaction questionnaire was given to the patient to analyse ADRs observed by patient. ADR assessment was done using WHO - UMC causality assessment scale, Hartwig - Siegel severity assessment scale and modified Schumock and Thornton preventability scale.

\section{Statistical Analysis}

Data were entered into the statistical software SPSS (version 20, developed by IBM) and analysis were performed accordingly. Descriptive statistics such as percentages were calculated for categorical variables. Mean and standard deviation were computed for continuous variables. Graphic representations were generated using Microsoft Excel 2007 for visual interpretation of analysed data.

\section{RESULTS}

Among 200 patients, 117 (58.5\%) were females and 83 $(41.5 \%)$ were males. Most of the patients were in the age group of $41-50$ years $(28.5 \%)$ and least in $0-10$ years $(2 \%)$ with mean age to be $49.32 \pm 14.069$ years. The prevalence of cancer in male patients of age 61-70 years was dominant, whereas cancer was most common in females of age group 41-50 years.

In 200 patients, 31 types of cancer were observed during the study period. Breast cancer $(22 \%)$ was the most common followed by stomach $(8.5 \%)$, lungs $(8 \%)$ and ovarian cancer $(8 \%)$.

The most prevalent cancer in females was breast cancer (37.61\%) followed by ovarian cancer $(13.67 \%)$ and multiple myeloma $(6.84 \%)$, whereas cancer of stomach $(15.66 \%)$ followed by lungs $(10.84 \%)$ and oesophagus $(9.64 \%)$ was most common in males.

Of the total number of patients, $19 \%$ were treated with monotherapy and remaining $81 \%$ were treated with combination drug regimen. Paclitaxel - Carboplatin $(11.11 \%)$ and Adriamycin - Cyclophosphamide (11.11\%) were the most frequently prescribed combination regimens.

Cyclophosphamide $(10.62 \%)$ was the most commonly prescribed anticancer drug in our study followed by oxaliplatin $(9.07 \%)$, paclitaxel $(8.85 \%)$, capecitabine $(7.96 \%)$, carboplatin $(6.64 \%)$ cisplatin $(6.19 \%)$, doxorubicin (5.75\%). These 7 drugs (cyclophosphamide, oxaliplatin, paclitaxel, capecitabine, carboplatin, cisplatin, doxorubicin) were considered as the most commonly prescribed drugs during the study period and were used to analyse ADRs in the study. Among 200 patients, 152 patients were administered the drugs that were most commonly prescribed in our study.

The most commonly prescribed anticancer drugs in male patients were oxaliplatin (13.20\%) followed by capecitabine $(12.18 \%)$, cisplatin (8.63\%), epirubicin $(6.60 \%)$ and in females was cyclophosphamide (14.12\%), followed by paclitaxel $(12.55 \%)$, carboplatin $(10.59 \%)$, doxorubicin $(8.63 \%)$.

Out of 152 patients who were administered the most commonly prescribed anticancer drugs as per the current study, adverse drug reactions were observed in 


\section{Table 1: Types of cancer.}

\begin{tabular}{|c|c|c|}
\hline SI. No & Type of Cancer & No. of Patients (Percentage) \\
\hline 1. & Breast & $44(22 \%)$ \\
\hline \multirow[t]{6}{*}{2.} & Head and neck: & $11(5.5 \%)$ \\
\hline & Buccal mucosa & $4(2 \%)$ \\
\hline & Tongue & $2(1 \%)$ \\
\hline & Oropharynx & $3(1.5 \%)$ \\
\hline & Maxilla & $1(0.5 \%)$ \\
\hline & Neck & $1(0.5 \%)$ \\
\hline \multirow[t]{2}{*}{3.} & Respiratory System & $16(8 \%)$ \\
\hline & Lungs & $16(8 \%)$ \\
\hline \multirow[t]{9}{*}{4.} & GIT : & $55(27.5 \%)$ \\
\hline & Esophagus & $13(6.5 \%)$ \\
\hline & Peritoneum & $1(0.5 \%)$ \\
\hline & Stomach & $17(8.5 \%)$ \\
\hline & Periampullary & $2(1 \%)$ \\
\hline & Hepatocellular & $3(1.5 \%)$ \\
\hline & Pancreas & $2(1 \%)$ \\
\hline & Rectal & $11(5.5 \%)$ \\
\hline & Colon & $6(3 \%)$ \\
\hline \multirow[t]{4}{*}{5.} & Brain : & $4(2 \%)$ \\
\hline & Glioblastomamultiforma & $2(1 \%)$ \\
\hline & Gemistocytic astrocytoma & $1(0.5 \%)$ \\
\hline & Medulloblastoma & $1(0.5 \%)$ \\
\hline \multirow[t]{2}{*}{6.} & Skeletal System : & $4(2 \%)$ \\
\hline & Ewings sarcoma & $4(2 \%)$ \\
\hline \multirow[t]{5}{*}{7.} & Genitourinary tract : & $28(14 \%)$ \\
\hline & Ovary & $16(8 \%)$ \\
\hline & Vagina & $9(4.5 \%)$ \\
\hline & Endometrium & $1(0.5 \%)$ \\
\hline & Testes & $2(1 \%)$ \\
\hline \multirow[t]{10}{*}{8.} & Others : & $38(19 \%)$ \\
\hline & Multiple myeloma & $13(6.5 \%)$ \\
\hline & ALL & $8(4 \%)$ \\
\hline & $\mathrm{NHL}$ & $6(3 \%)$ \\
\hline & AML & $3(1.5 \%)$ \\
\hline & $\mathrm{HL}$ & $2(1 \%)$ \\
\hline & CML & $3(1.5 \%)$ \\
\hline & Leiomyosarcoma & $1(0.5 \%)$ \\
\hline & Adenocarcinoma, unknown origin & $2(1 \%)$ \\
\hline & TOTAL & $200(100 \%)$ \\
\hline
\end{tabular}

109 patients with incidence of $71.71 \%$. Among these $39.45 \%$ were male patients and $60.55 \%$ were females. Female patients had a higher incidence of ADR than male patients. Adverse drug reactions observed for the most commonly prescribed anticancer drugs is listed in Table 2 .

The most frequent adverse reaction was vomiting $(10 \%)$ followed by anaemia $(9.11 \%)$, alopecia $(7.11 \%)$ and fatigue $(6.67 \%)$. The most common ADRs in male patients was vomiting $(9.66 \%)$ followed by anaemia $(8.28 \%)$, fatigue $(7.59 \%)$, diarrhoea $(6.90 \%)$ and that in female patients was vomiting $(10.16 \%)$ followed by anaemia $(9.51 \%)$, alopecia $(8.85 \%)$, fatigue $(6.23 \%)$. Gastrointestinal adverse reactions were most frequent followed by haematological adverse effects.

The causal relationship of ADR with the drug was analyzed using WHO - UMC causality assessment scale. Among 450 ADRs observed, 4 (0.89\%) were certain, 182
(40.44\%) were probable, $206(45.78 \%)$ were possible and $58(12.89 \%)$ were unlikely. Adverse reactions that are said to be certain include capecitabine induced vomiting, diarrhoea and paclitaxel induced rashes, pruritis.

On assessing the severity of ADRs by Hartwig Siegel severity assessment scale, $59(54.13 \%)$ patients experienced mild ADR, whereas $49(44.95 \%)$ patients showed moderate level of ADR and $1(0.92 \%)$ patient experienced severe level of ADR from the total of 109 patients.

Preventability assessment of ADRs was analysed using Schumock - Thornton preventability assessment scale. Out of 450 ADRs observed, 208 (46.22\%) were definitely preventable, $93(20.67 \%)$ were probably preventable and $149(33.11 \%)$ ADRs were not preventable. ADRs like nausea, vomiting, decreased blood counts, fever were definitely preventable. Reactions like diarrhoea, 
Table 2: Observed adverse reactions of most commonly prescribed anticancer drugs.

\section{MOST COMMONLY \\ PRESCRIBED \\ ANTICANER DRUGS}

Cyclophosphamide

Oxaliplatin

Capecitabine

Carboplatin

Cisplatin

Doxorubicin

\section{OBSERVED ADVERSE DRUG REACTIONS}

Anorexia, fatigue, diarrhoea, vomiting, nausea, infection, leukopenia, neutropenia, alopecia, head ache, mucositis, altered taste, nail discolouration, burning sensation, abdominal pain, fever, thrombocytopenia, anaemia, hypochloremia, hyponatremia, urinary retention, dyspnoea, rashes, abdominal bloating, dysuria, skin peeling, abnormal urea, swollen face, pedal edema.

Anaemia, neutropenia, vomiting, diarrhoea, fatigue, leukopenia, abdominal pain, nausea, anorexia, constipation, weight loss, parasthesia, mucositis, head ache, cough, thrombocytopenia, itching, dyspnoea, burning sensation, fever, back pain, dysuria.

Fatigue, alopecia, anaemia, dyspnoea, rashes, pruritis, back pain, leg pain, infection, body pain, vomiting, parasthesia, neuropathy, neutropenia, altered taste, nausea, muscle pain, leukopenia, mucositis, pedal edema, diarrhoea, cough, swollen face, sweating, thrombocytopenia, infection, sinus tachycardia, abdominal pain, anorexia, shoulder pain.

Vomiting, anaemia, neutropenia, diarrhoea, fatigue, leukopenia, nausea, anorexia, abdominal pain, HFS, skin peeling, mucositis, head ache, cough, thrombocytopenia, constipation, fever, chills, dysuria.

Rashes, alopecia, anaemia, fever, chills, pruritis, back pain, vomiting, fatigue, parasthesia, neuropathy, neutropenia, hematuria, abdominal pain, muscle pain, nausea, leukopenia, increased LFT, pedal edema, diarrhoea, body pain, thrombocytopenia, hypochloremia, hypokalemia, tooth pain, shoulder pain, hyponatremia.

Anaemia, vomiting, nausea, hypomagnesemia, hypokalemia, leukopenia, thrombocytopenia, hyponatremia, alopecia, rashes, fever, anorexia, hypochloremia, muscle pain, neutropenia, swollen face, dysuria, dyspnoea, body pain, hematuria, increased LFT.

Mucositis, fever, neutropenia, leukopenia, nausea, rashes, nail discolouration, alopecia, fatigue, vomiting, infection, muscle pain, anorexia, chills, sinus tachycardia, hematuria, weight loss, abdominal guarding and rigidity, tooth pain.

constipation, and mucositis were probably preventable and rest of the ADRs were considered to be not preventable.

\section{DISCUSSION}

Current study findings revealed that combination drug regimen was frequently prescribed to the patients than single drug regimen for a better therapy. The most commonly prescribed combination drug regimen was paclitaxel - carboplatin and adriamycin cyclophosphamide. This is coincidental to the findings of Chan, et al ., (2014) and Afzal, et al ., (2014) which showed paclitaxel - carboplatin (36.7\%) and AC (4.61\%) respectively to be the most commonly prescribed chemotherapeutic regimen. ${ }^{4,5}$

Among the total chemotherapeutic agents prescribed in our study, cyclophosphamide was the most common agent followed by oxaliplatin, paclitaxel, capecitabine, carboplatin, cisplatin, doxorubicin. This was almost similar to the study conducted by Thennarasu, et al ., which revealed that paclitaxel $(36.6 \%)$ to be the most commonly prescribed drug followed by capecitabine $(27.5 \%)$, cyclophosphamide $(22.9 \%)$, cisplatin $(18.30 \%)$, doxorubicin $(15.5 \%)$, carboplatin $(13.70 \%)$, oxaliplatin $(5.50 \%){ }^{6}$

The drugs which were most commonly prescribed in our study were used for further analysis such as determination of adverse drug reactions. These drugs belongs to the class of antineoplastic agents like alkylating agents (nitrogen mustards, platinum coordinating complexes), antimetabolites (pyrimidine analogs), taxanes, antitumor antibiotics. Studies conducted by Mallik, et al ., (2007) and Kaur, et al ., (2015) ${ }^{8}$ reported alkylating agents, antimetabolites, antitumor antibiotics, taxanes as the drugs causing ADR.

The demographic profile of present study shows that majority of females were found to have ADRs than males with the incidence of ADR to be $71.71 \%$. This is consistent with another study done by Chopra, et al ., (2016) which showed incidence of ADR to be $58.6 \%$, presence of ADR in males to be $26.4 \%$ and females $73.6 \%{ }^{9}$ Adverse drug reactions are known to occur commonly in female population than the males.

In the present study the most frequent adverse reaction was vomiting followed by anaemia, alopecia, fatigue. This is complementary to the findings of Chopra, et al., (2016) which revealed nausea, vomiting $(25.5 \%)$ as the most common ADR followed by alopecia (20.9\%), leucopenia (18.6\%). ${ }^{9}$ The frequency of alopecia $(27.76 \%)$ followed by anaemia $(7.48 \%$ ) was higher in a study done by Kaur, et al ., (2015). ${ }^{8}$ This was contrary to the findings of Sharma, et al ., (2015) which showed infection as the major ADR. ${ }^{3}$ The most common mechanism of chemotherapy induced nausea and vomiting may be activation of CTZ (Chemoreceptor trigger zone). Since 
vomiting is a common problem associated with cancer chemotherapy, strategies should be made to prevent and manage vomiting in patients undergoing therapy. While destroying cancer cells, chemotherapy can also damage rapidly dividing cells of bone marrow resulting in myelosuppression by affecting WBCs, platelets and RBCs.

The most common ADRs in male patients was vomiting followed by anaemia, fatigue, diarrhoea and that in female patients was vomiting, followed by anaemia, alopecia, fatigue in this study. This was contrary to the findings of Bellare, et al ., (2016) who showed hyponatremia $(12 \%)$ and infection $(9 \%)$ as the most common ADR for males and hyponatremia ( $9.8 \%)$, neutropenia $(11.8 \%)$ in females. ${ }^{10}$ The occurrence of neutropenia was very less when compared to other studies. Gastrointestinal and haematological ADRs were frequently observed in the current study which resembles the study done by Thennarasu, et al., (2015). ${ }^{6}$

In the current study, based on WHO - UMC causality assessment scale, it was found that most of the ADRs were under the category of possible followed by probable, unlikely, and certain. This was analogous to the findings of Thennarasu, et al ., (2015) which found out most of the ADRs to be possible $(70.50 \%)$ followed by probable $(25.90 \%)$, unlikely $(3.35 \%)$ and certain $(0.33 \%){ }^{6}$ Severity of ADRs was assessed using Hartwig - Siegel severity assessment scale in our study, which revealed most of the ADRs to be mild, moderate and severe. A study conducted by Swathi, et al., (2015) contradicts our findings by reporting moderate $(69 \%)$ level of ADRs as highest followed by mild $(12 \%)$ and severe $(19 \%) \cdot{ }^{[1]}$ The preponderance of mild level $(65.90 \%)$ of ADRs was higher than moderate $(34.86 \%)$ in a study done by Thennarasu, et al ., (2015). ${ }^{6}$

Preventability of adverse reactions was assessed in our study using modified Schumock and Thornton scale which showed $46.22 \%$ of ADRs was definitely preventable, $20.67 \%$ were probably preventable and $33.11 \%$ were not preventable. This is similar to the findings of Swathi, et al., (2015) that did ADR assessment of commonly used platinum compounds and showed $48 \%$ of reactions were definitely preventable, $16 \%$ were probably preventable and $36 \%$ were not preventable. ${ }^{11}$ Majority of the drugs used to assess ADRs in our study belongs to the class of platinum coordinating complexes. On the contrary, preventability assessment made by Chopra, et al ., (2016) reported as half $(51 \%)$ of ADRs as not preventable, $42 \%$ probably preventable and $7 \%$ definitely preventable. ${ }^{9}$ In our study ADRs like nausea, vomiting, decreased blood counts, fever were considered to be definitely preventable. Reactions like diarrhoea, constipation, and mucositis were probably preventable and rest of the ADRs were considered to be not preventable. Although an adequate premedication with parenteral dexamethasone, ranitidine and ondansetron were given to each patient, the frequency of vomiting remained high due to high emetogenic potential of chemotherapeutic agents. ${ }^{11}$

The study was conducted for a very short period of time, hence the sample size was also less which is a major limitation. ADR questionnaire was given to the patient on the review date after at least one cycle of chemotherapy, lack of patients who visited hospital on correct review date was also a limitation.

\section{CONCLUSION}

Most of the anticancer drugs were prescribed in combination for a better therapy. Among 41 anticancer drugs, cyclophosphamide was the most commonly prescribed. Chemotherapy has a high potential to cause ADR in cancer patients. Most frequently observed ADRs were gastrointestinal and haematological reactions. Most common adverse effect was vomiting. Vomiting can be preventable, so there is a need to improve the management of vomiting, since the rates of prevention of these adverse effects were poor. In the present study, causality of adverse effects was poor due to the presence of co administered drugs.

Since cancer chemotherapy has a high potential to cause ADRs, measures need to be put into place to reduce the physical, emotional and economic burden on the patient due to adverse drug reactions. There is a need for vigilant ADR monitoring to decrease morbidity and mortality due to ADRs, which requires further studies on larger population. This study demonstrated that monitoring of chemotherapy related ADRs are feasible in a cancer ward and can facilitate quality improvement initiatives, as well as potentially improve patient care. Measures to improve detection and reporting of ADRs should be taken to enhance our understanding the nature and impact of these ADRs. By implementing the ADR monitoring and reporting system, pharmacists can promote drug safety and thereby assist health care professionals for a better patient care.

\section{ACKNOWLEDGEMENT}

Our sincere gratitude to all the faculties of medical oncology department of Father Muller Medical College, Mangalore. Above all, we remain indebted to the most courageous people with cancer in our study for their cooperation.

Indian Journal of Pharmacy Practice, Vol 10, Issue 4, Oct-Dec, 2017 


\section{CONFLICT OF INTEREST}

The authors hereby declare no conflict of interest.

\section{ABBREVIATIONS USED}

UMC: Uppsala Monitoring Center; ALL - Acute lymphoblastic leukemia; NHL- Non Hodgkins lymphoma; AML: Acute myeloid leukemia; HL- Hodgkins lymphoma; CML: Chronic myeloid leukemia.

\section{SUMMARY}

Periodic assessment of ADR on anticancer drugs is highly essential as its impact on patients with chemotherapy is severe. ADR monitoring process promotes better prescribing habits in patients for safe and effective use of chemotherapeutic agents. This could improve the quality of life in cancer patients.

\section{REFERENCES}

1. WHO International drug monitoring the role of hospitals technical report series, WHO, Geneva, (1969):425.

2. Saini VK, Sewal RK, Ahmad Y, Medhi B. Prospective observational study of adverse drug reactions of anticancer drugs used in cancer treatment in a tertiary care hospital. Indian Journal of Pharmaceutical Sciences. 2015;77(6):687-93.
3. Sharma A, Kumari KM, Manohar HD, Bairy KL, Thomas J. Pattern of adverse drug reactions due to cancer chemotherapy in a tertiary care hospital in South India. Perspectives in Clinical Research. 2015;6(2):109-15.

4. Chan HK, Ismail S. Side effects of chemotherapy among cancer patients in a Malaysian general hospital: experiences, perceptions and informational needs from clinical pharmacists. Asian Pacific Journal of Cancer Patients. 2014;15(3):5305-9.

5. Kirthi C, Afzal A, Reddy M, Ali SA, Yerramilli A, Sharma S. A study on the adverse effects of anticancer drugs in an oncology centre of a tertiary care hospital. International Journal of Pharmacy and Pharmaceutical sciences. 2014;6(2):580-3.

6. Thennarasu P, Ramesh A, Rani V, Vemullapalli S, Kannan G. Incidence of adverse drug reactions in patients on cancer chemotherapy in a tertiary care teaching hospital. Research Journal of Pharmaceutical, Biological and Chemical sciences. 2015;6(5):214-26

7. Mallik S, Palaian S, Ojha P, Mishra P. Pattern of adverse drug reactions due to cancer chemotherapy in a tertiary care teaching hospital in Nepal. Journal of pharmaceutical Sciences. 2007;20(3):214-8.

8. Kaur K, Sood M, Bhagat S, Singh T, Jain M, Arora D, et al. Spontaneous adverse drug reaction monitoring in oncology: our experience. Indian Journal of cancer. 2015;52(3):467-70.

9. Chopra D, Rehan HS, Sharma V, Mishra R. Chemotherapy induced adverse drug reactions in oncology patients: a prospective observational survey. Indian Journal of Medical and Paediatric oncology. 2016;37(1):42-6.

10. Bellare PS, Ashwin K, Prakash S, Vinaykumar S, Rakesh KB. A retrospective evaluation of adverse drug reactions due to cancer chemotherapy in a tertiary care hospital in South India. Journal of young pharmacists. 2016;8(3):251-4.

11. Swathi B, Bhavika D, Begum N. Adverse drug reaction profiles of commonly used platinum compounds in cancer chemotherapy. International Journal of Basic and Clinical Pharmacology. 2015;4(2):284-9. 